\section{Commentary: The Cancer Care Delivery System in the 00s}

In this new JNCCN feature, experts will address issues and programs that will influence the delivery system for cancer care over the next decade. Individual submissions for this new section of JNCCN are most welcome.

The return of substantial rates of rise in national health care expenditures, health insurance premiums, and employer contributions is beginning to make the current environment feel a lot like the health care system in the late 1980s and early 1990s. In those days, rising health care costs brought tremendous tension and pressure to bear on both employers and patients in their roles as purchasers of care. These pressures were translocated to health insurers, who were charged to reduce the pressures and, thus, sought to morph into companies that could manage health care. Hence, a managed care industry emerged.

Managed care companies did have some modest and transient success in moderating the rate of rise of health care expenditures in the mid to late 1990s. The one successful mechanism used to accomplish this was a focus on aggressively negotiating down the reimbursement for services provided by facilities and by professionals. Managed care companies used the specter of provider exclusion of from "narrow networks" to extract pricing concessions.

These pricing concessions from providers were achieved in the early years of the 1990s, when managed care companies rode the crest of power. However, that power began to recede in 1995. The Clinton Health Care Reform debate of 1994 provided one conclusive result, and that was the affirmation of the need for "choice" for patients and their families in deciding the most appropriate and effective provider and provider setting for the delivery of the care they needed. Employers, as direct influencers of the "product lines" of managed care companies, embraced the concept of "choice." Thus, the leverage carried by the threat of narrow networks began to diminish for managed care companies in the negotiation and quest for ever-lower reimbursement for provider services.

What did not diminish was the steady and increasing flow of innovative technologies (drugs, devices, procedures, and techniques) into the health care system. Many of these technologies offer advantages in safety and effectiveness. Many also come with a higher price tag and, in some instances, are used in concert with existing standard clinical interventions. Thus, although the negotiating power of the managed care companies provided a brief respite from accelerating health care costs, the reality of demand for consumer choice, regrouping of the provider side, and infusion of new technology resulted in a predictable, strong resurgence in health care pricing pressures.

Cancer care was at the forefront of coverage and reimbursement issues in the 1990s and will find itself positioned similarly in this new millennium. This is because the oncology research enterprise has pipelines replete with promising new drugs and biologic agents designed for disease states that are serious and life-threatening. The inadequacies of existing interventions for some cancers are being addressed incrementally and, in a few cancers, substantially, with the introduction of new diagnostics and treatments. The demand for such innovative technologies is strong from patients, providers, and society as a whole. Additionally, the lower toxicity profiles of new agents will convert the

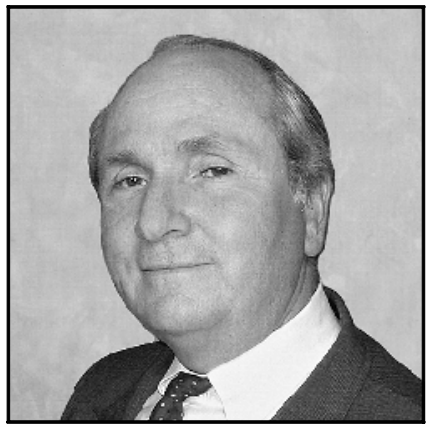

William T. McGivney, PhD

William T. McGivney, PhD, is the Chief Executive Officer of the National Comprehensive Cancer Network and a recognized expert in coverage policy and drug and device regulatory policy. Before joining the NCCN in 1997, Dr. McGivney directed the Division of Health Care Technology at the American Medical Association and worked for Aetna Health Plans as Vice President for Clinical and Coverage Policy. Awarded the FDA Commissioner's Medal of Appreciation in 1989, Dr. McGivney has served on numerous national boards and committees. He earned his PhD at the University of North Carolina at Chapel Hill and completed a postdoctoral fellowship in the Department of Psychiatry at the Harvard Medical School.

Call for Commentaries

JNCCN welcomes commentaries discussing issues in cancer care delivery in the next decade as well as on topics relating to cancer prevention, detection, treatment, supportive care, or survivorship from a public health policy perspective. Articles should be no more than 1500 words (approximately 6-8 typed, double-spaced pages). For more information, to check on topic suitability, or to submit a commentary, please contact:

Managing Editor

JNCCN

500 Old York Road, Suite 250 Jenkintown, PA 19046 Phone: 215-690-0235

Fax: 215-690-0281 (attn: JNCCN) E-mail: callan@nccn.org 
therapeutic strategies for some cancers into ones similar to those for chronic diseases. The advantages in therapeutic index also will be accompanied by higher price tags. Additionally, the costs of these new agents will be magnified by the ability to be included in multiple and consecutive regimens and by the ability to be administered over longer periods of time (until disease progression). The advent of many of these agents is at hand.

Given the urgency of the situation, what is the likely response of the employer and payor communities? The strategy is fourfold, and the emphasis will likely vary with the size and sophistication of the care manager. First, there is talk about returning to the confrontational days of coverage restrictions, medical necessity denials, preauthorization requirements, and even retrospective review. Second, the system will seek to involve the patient as consumer more in decisions about provider, setting for care delivery, treatment, and financial contribution. Third, the call for reporting performance data from providers, especially institutions, is growing. Finally, the concept of "pay for performance" is being discussed as a mechanism to enhance the efficiency of health care delivery.

In this commentary, I focus on the first strategy above. Subsequent commentaries will discuss the application and implementation of some of the more forward-thinking positions being advanced by various constituencies in health care.

\section{Health Care Decision-Making Redux: Coverage Policy and Medical Necessity}

Studies about significant variation and about inappropriate use of health care technologies were easy to find in the late 1980s. Management of variation and overuse were viewed as ways to improve effectiveness and to control costs. Managed care companies adopted strategies to exert control over these issues. These strategies were translated into programs of detailed and circumscribed coverage policies and implementation of coverage policies through medical necessity determinations, preauthorization requirements, and, less often, retrospective review. The operation of the programs achieved very modest results, usually in areas of gross, inappropriate overuse. The programs also resulted in a tremendous inefficiency in the use of administrative resources on both the payor and the provider side, and more importantly, delays or denials of access to needed care for patients. This was particularly true in serious and life-threatening diseases like cancer, for which the exigencies of the seriously ill and the hope offered by new technologies tend to converge. It is disconcerting to hear now of the plans of a new generation of managed care medical directors who, unfamiliar with the fruitless battles of the 1990s, view the aforementioned efforts as reasonable ways to deal with decisions about using innovative technologies.

Clearly, many constituencies in the health care system have recognized the need to better integrate evidence-based decision-making into the overall health care policy process. The possibility that the contentious days of the 1990s may be revisited with the increased emphasis on programs (such as medical necessity determinations) that can intrude into reasonable decisions made between physicians and patients is of great concern, especially in talking about cancer care.

Were no lessons learned? Indeed, I have written that there is a major and inappropriate disconnect between clinical and coverage decision-making processes. 
Clinical decision-making clearly constitutes a risk-benefit analysis. Coverage decision-making should really be a parallel process. Indeed, when one gets down to the interpretation of individual patient needs in a medical necessity determination, one really is involved in the practice of medicine. In that sense, coverage decision-making as the basis for medical necessity determinations should also involve a risk-benefit analysis. However, the establishment of coverage policy tends to be a binary (yes/no) process, which is not flexible enough to consistently drive decisions (such as medical necessity determinations) tailored to individual patient needs.

This circumstance is exacerbated for the seriously ill cancer patient. Serious or life-threatening illness demands reasoned flexibility in therapeutic decisionmaking. Indeed, in life-threatening conditions, physicians, patients, and payors should be willing to accept a lesser degree of certitude about effectiveness and a greater risk of harm in decisions about therapeutic interventions. When a managed care decision-maker is abiding by a strict interpretation of written contractual language and written coverage policy, then integration of thoughtful risk-benefit considerations is difficult if not impossible.

A return by managed care companies to the intrusive processes of the 1990s will not enhance the quality or efficiency of cancer care. Rather, we must move to a system in which decisions proceed from guidelines based on sound scientific evidence integrated with expert opinion. Notwithstanding the idealists, the integration of expert opinion, with efforts to minimize bias, is necessary given the many situations for which we have insufficient evidence or no evidence at all. In developing such recommendations, the basis for the recommendations should be explicitly stated.

The application of guidelines facilitates a formal, structured consideration of available options and explicit identification and delineation of resources consumed. In the longer term, performance measurement must be included as a critical component of a system of continuous quality improvement. The system should seek to improve care based on analysis, feedback, and action on data that describe practice patterns, the level of concordance of such practice with recognized guidelines, and the outcomes achieved by such practice. The final step is a defined process by which to act on the feedback to effect change in and improve the effectiveness and efficiency of patient care.

\section{Bottom Line}

Hopefully, we can leave the aforementioned intrusive mechanisms of use control behind. Now is the time to consider the more enlightened proposals emanating from many constituents of the health care system. At present, sophisticated employers and business coalitions are actively pursuing such change. The recent passage of the Medicare Prescription Drug, Improvement and Modernization Act presages a move by Medicare in the same direction. In cancer care, many organizations and institutions have established programs that seek to measure, evaluate, and improve the care that is being provided. These efforts are laudable and will result in improved care.

In the next Commentary, we will take the next step (a large one indeed) and address the call for the public reporting of performance measures and the implications of these for the cancer care delivery system. 